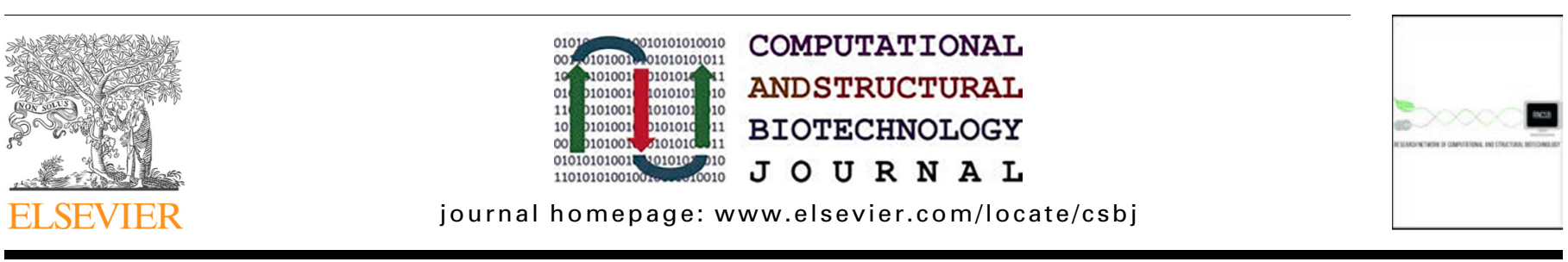

\title{
The impact of Gag non-cleavage site mutations on HIV-1 viral fitness from integrative modelling and simulations
}

\author{
Firdaus Samsudin ${ }^{\mathrm{a}}$, Samuel Ken-En Gan ${ }^{\mathrm{a}, \mathrm{c}, \mathrm{d}, *}$, Peter J. Bond ${ }^{\mathrm{a}, \mathrm{b}, *}$ \\ a Bioinformatics Institute (A*STAR), 30 Biopolis Street, \#07-01 Matrix, Singapore 138671, Singapore \\ ${ }^{\mathrm{b}}$ Department of Biological Sciences, National University of Singapore, 14 Science Drive 4, Singapore 117543, Singapore \\ ${ }^{\mathrm{c}}$ Antibody \& Product Development Lab - Large Molecule Innovation, Experimental Drug Development Centre (A*STAR), 138670 Singapore, Singapore \\ d 553 Laboratory (A*STAR), 138648 Singapore, Singapore
}

\section{A R T I C L E I N F O}

\section{Article history:}

Received 29 September 2020

Received in revised form 15 December 2020

Accepted 16 December 2020

Available online 23 December 2020

\section{Keywords:}

Protease inhibitor drug resistance

HIV-1

Group-specific antigen (Gag)

Integrative modelling

Multiscale simulation

\begin{abstract}
A B S T R A C T
The high mutation rate in retroviruses is one of the leading causes of drug resistance. In human immunodeficiency virus type-1 (HIV-1), synergistic mutations in its protease and the protease substrate - the Group-specific antigen (Gag) polyprotein - work together to confer drug resistance against protease inhibitors and compensate the mutations affecting viral fitness. Some Gag mutations can restore Gagprotease binding, yet most Gag-protease correlated mutations occur outside of the Gag cleavage site. To investigate the molecular basis for this, we now report multiscale modelling approaches to investigate various sequentially cleaved Gag products in the context of clinically relevant mutations that occur outside of the cleavage sites, including simulations of the largest Gag proteolytic product in its viral membrane-bound state. We found that some mutations, such as G123E and $\mathrm{H} 219 \mathrm{Q}$, involve direct interaction with cleavage site residues to influence their local environment, while certain mutations in the matrix domain lead to the enrichment of lipids important for Gag targeting and assembly. Collectively, our results reveal why non-cleavage site mutations have far-reaching implications outside of Gag proteolysis, with important consequences for drugging Gag maturation intermediates and tackling protease inhibitor resistance.
\end{abstract}

(c) 2020 The Author(s). Published by Elsevier B.V. on behalf of Research Network of Computational and Structural Biotechnology. This is an open access article under the CC BY-NC-ND license (http://creativecommons.org/licenses/by-nc-nd/4.0/).

\section{Introduction}

RNA viruses are among the most adaptable pathogens threatening human health. With their high mutation rates [1] they are orders of magnitude more adaptable than most DNA-based organisms [2] resulting in their ability not only to escape the immune system and become drug resistant, but jump across host species boundaries causing emerging infectious diseases that could turn into global pandemics [3-6]. HIV-1 represents one of the most extensively characterized RNA viruses, with mathematical modelling showing that many clinically established drug resistance mutations could occur within the first replication cycle [7] and every single possible point mutation could occur thousands of times within a single day, leading to rapid drug resistance [8] hence leading to the need to treat HIV patients with a cocktail of

* Corresponding authors at: Bioinformatics Institute (A*STAR), 30 Biopolis Street, \#07-01 Matrix, Singapore 138671, Singapore.*

E-mail addresses: samuel_gan@eddc.a-star.edu.sg (S.K.-E. Gan), peterjb@bii.astar.edu.sg (P.J. Bond). multiple antiviral drugs with different mechanisms [9]. Understanding the role of these mutations is thus an imperative aspect in novel antiviral drug development.

Most clinically approved HIV treatments target various key enzymes that are critical to the life cycle of the virus, one of which is the HIV-1 protease. HIV-1 protease cleaves the structural polyproteins Gag and Gag-Pol into their mature components to create the infectious virion. To inhibit this, protease inhibitors (PIs) bind competitively to the viral protease to prevent Gag substrate proteolysis and virion maturation. PIs form a vital second line component of the antiretroviral therapy (ART) in the management of adults with HIV. As failure of first line regimens develop, a potent second line drug is crucial [10]. This is often associated with multi nucleoside reverse transcriptase inhibitors (NRTIs) resistance. Ritonavir-boosted PIs represents the only available secondline treatment option for the majority of HIV patients worldwide; however, emerging mutations in HIV-1 protease often render PIs ineffective, with some mutations even leading to cross-drug resistance [11-13]. This is further aggravated by synergistic mutations on the Gag polyprotein itself [14-17] which is the rationale for 
elucidating the drug resistance encoded in Gag. Gag mutations around the protease cleavage site have been shown to restore Gag-protease binding by introducing new chemical interactions and inducing subtle conformational changes that compensate for the loss of affinity due to the mutations on HIV-1 protease [18]. Apart from mutations around the protease cleavage sites, Gag also harbours mutations away from these regions, many of which have been shown to directly contribute towards PI resistance [19-21]. To date, the role of these non-cleavage site mutations is largely unknown, with some analysis pointing to allosteric communication [22]. A study using paramagnetic NMR spectroscopy showed that Gag can form transient complexes with protease and the binding interface involves non-cleavage site regions that are prone to mutation causing drug resistance [23]. In this study, we aim to use structural modelling and simulations to decipher how Gag non-cleavage site mutations contribute towards the overall fitness of the HIV-1 virus.

The HIV-1 Gag polyprotein is a 500 amino acid precursor protein containing the matrix (MA), capsid (CA), nucleocapsid (NC) and P6 domains, as well as two spacer peptides, SP1 and SP2. During the late phase of the HIV-1 replication cycle, Gag is sequentially cleaved by the protease enzyme into these domains (Fig. S1), which subsequently form an infectious mature virion [24]. Each domain plays a specific role during maturation; for example, the MA domain drives full-length Gag to assemble at the plasma membrane of the host cell, while the CA and NC domains encapsulate the viral RNA genome. The CA domain also interacts with CypA, which is a host cell protein that is incorporated into the virion and is essential for capsid uncoating [25,26]. Due to its functional significance in the HIV-1 life cycle, Gag is an attractive target for therapeutic agents and to date, several drugs that target the CA domain have been identified [27]. Nevertheless, a Gag inhibitor is yet to be clinically approved. For example, Bevirimat is a drug candidate that stabilises the immature Gag lattice by preventing proteolysis between the CA and SP1 domains [28]. A clinical trial, however, showed a reduced response amongst certain patients due to the highly polymorphic nature of Gag across different circulating HIV-1 subtypes and various resistant mutations [29,30] which highlights the importance of understanding the role of Gag non-cleavage site mutations.

Over the past few years, integrative modelling and molecular dynamics (MD) simulations, in tandem with advances in structural biology, have provided valuable insights into the molecular mechanism underlying viral function and dynamics [31]. In the study of HIV-1, this ranges from multiscale simulations of the entire viral capsid shell and its assembly pathways [32-37] to simulations of individual Gag proteins with host cell components, such as the CA domain with CypA [38] kinesin-1 adaptor protein FEZ1 [39] and inositol hexakisphosphate (IP6) [40] as well as the MA domain with a model plasma membrane [41]. A previous structural model of full-length monomeric HIV-1 Gag revealed allosteric communications between non-cleavage site mutations and the first Gag cleavage site [22] providing a glimpse into how residues far away from the protease cleavage site could affect proteolysis. However, this model did not take into account in vivo Gag oligomerisation, which is instrumental during virus particle maturation. Crystal structures show that the MA domain exists as a trimer [42] whilst the CA domain forms a hexamer [43]. An electron microscopy (EM) study of the MA protein in a PIP2 containing membrane showed that under higher order conditions, it organizes into hexamersof-trimers [44].

Structural models of sequentially cleaved Gag are imperative for understanding the conformational changes involved upon proteolysis, which may improve our overall knowledge of Gag mutations and potential drugging of these intermediates. Using available structures of HIV-1 Gag domains, we built an integrative model of the complete oligomeric Gag polyprotein cleavage product (Fig. 1) bound to a viral membrane model (Fig. 2). Based on coarse grained (CG) MD simulations using the Martini forcefield of the wild-type (WT) and mutant Gag variants, supported by careful calibration against atomic-resolution sampling, we found that non-cleavage site mutations can interact with cleavage site residues and potentially alter their local environment, while mutations on the MA domain confer stronger binding to the plasma membrane. Subsequent all-atom MD simulations of the CA domain revealed how mutations in this region modulate CypA interaction as well as stabilise oligomer formation. Overall, our study uncovers how these distant mutations can affect various processes during HIV-1 viral maturation and contribute towards its overall fitness.

\section{Results}

\subsection{Membrane dynamics of the HIV-1 Gag MA-CA-SP1 polyprotein}

The Gag polyprotein is sequentially cleaved by HIV-1 protease during viral maturation (Fig. S1). It is unknown which state of viral maturation is affected by non-cleavage site mutations. As such, we aim to investigate the effects of these mutations on both immature and mature states of Gag, where structures are available. The first cleavage occurs between the SP1 and NC domains to produce the MA-CA-SP1 polyprotein, which represents the largest Gag cleavage product. A hexameric model of the HIV-1 Gag MA-CA-SP1 polyprotein was built using the crystal structures of the MA trimer [42] and CA hexamer [43] (see Methods section for further details) (Fig. 1). The crystal structure of the immature CA hexamer was aligned with six copies of MA trimers (hexamers-of-trimers) following the arrangement observed in membrane bilayers [44]. A solution NMR structure of an N-terminal fragment of immature HIV-1 Gag that includes MA and the C-terminal portion of CA shows that the linker between MA and CA domains is inherently flexible and adopts multiple conformation [45]. This is supported by another NMR study of an HIV-1 Gag fragment consisting of the MA, CA, SP1 and NC domains that showed individual domains orient in a manner semi-independent of each other, with adjoining linkers that are intrinsically disordered [46]. As such, we constructed a flexible intervening linker between the MA and CA domains $a b$ initio. Gatanaga et al. discovered PI-resistant Gag variants with non-cleavage site mutations mapped to the MA-CA-SP1 complex (Table S1, Fig. S1) [20]. These include seven unique mutations, with five located in the MA domain and two in the CA domain. We generated models for three variants (specifically variant numbers 3, 6, and 7, as outlined in Table S1) that cover all of these seven unique mutations (Fig. 2A). These models were built by substituting the wild-type residues with the corresponding mutant version, assuming that these point mutations do not have any large structural impact upon Gag. To understand the conformational dynamics of the polyprotein and the potential roles of these non-cleavage site mutations, we performed four independent $15 \mu$ s CG MD simulations of the MA-CA-SP1 model bound to a realistic HIV-1 membrane model (Simulations 1-4 in Table S2). The membrane model contained phosphatidylcholine (PC), phosphatidyl ethanolamine (PE) and phosphatidylserine (PS) lipids, as well as PIP2, sphingomyelin and cholesterol, as determined by a previous lipidomics study [47] (see Methods section for further details), while the MA domain $\mathrm{N}$-terminus was myristoylated (Fig. 2B).

As previously predicted [41] the myristate group on the $\mathrm{N}$ terminal glycine residue of MA domain effectively anchored the protein to the membrane. Within the first few nanoseconds, the linker connecting the MA and CA domains contracted, effectively pulling the CA-SP1 domain towards the membrane (Fig. 2B). To 
A

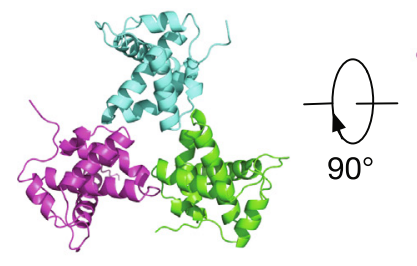

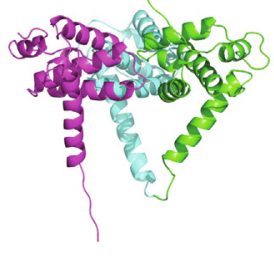

B

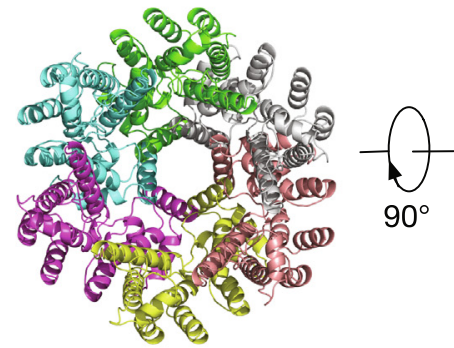

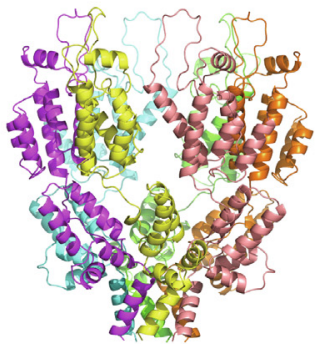

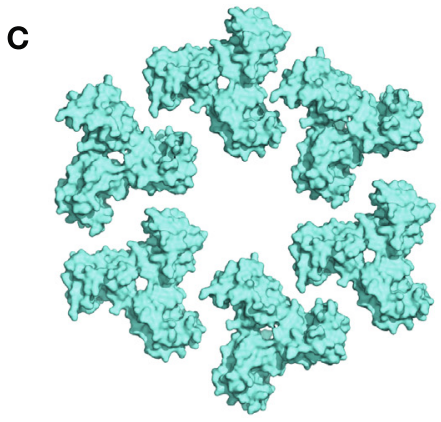

D
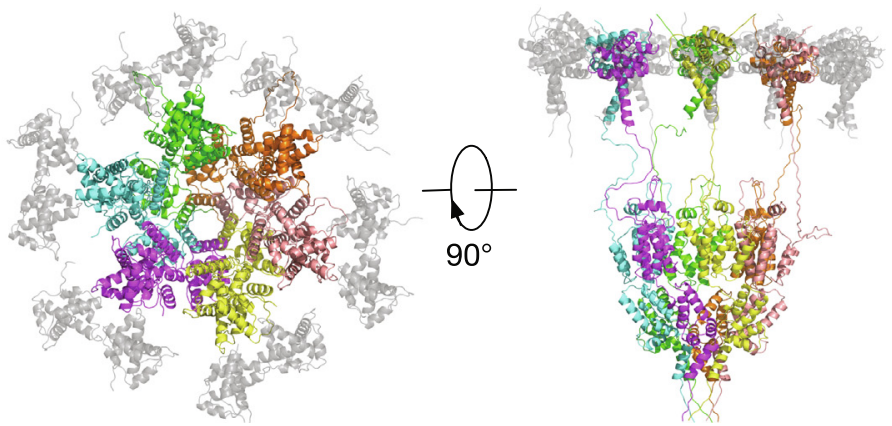

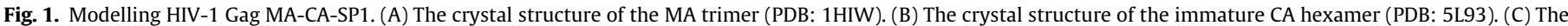

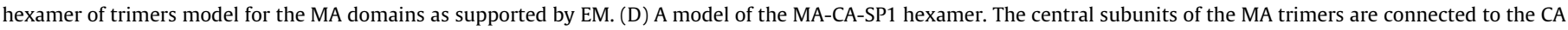
domains and coloured as in (B), whereas the peripheral MA subunits are coloured grey.

verify the MA-CA linker contraction observed during CG sampling, we also performed four independent $500 \mathrm{~ns}$ atomistic simulations of the MA-CA-SP1 monomer with positional restraints applied to the backbone atoms of lipid binding residues to mimic membrane association (Simulation 5 in Table S2). Additionally, we conducted CG simulations of the same monomeric system (Simulation 6 in Table S2). Both of these additional sets of simulations showed a contraction of the MA-CA linker, resulting in a similar distribution of distances between the MA and CA domains compared to the CG hexamer simulations (Fig. S2). While the atomistic simulations initially sampled a wider spread of distances, after around 300 ns this converged to 4-6 $\mathrm{nm}$, reproducing the CG distributions. This validates our CG hexamer model and shows that the linker region does not maintain an extended conformation, but rather contracts to allow direct contact between the MA and CA domains.

Curvature has been shown to be important in modulating the interface of the CA lattice $[43,48]$. Structural flexibility of the CA $\mathrm{N}$-terminal and $\mathrm{C}$-terminal domains give rise to a curved $\mathrm{CA}-\mathrm{CA}$ interface, and oligomerisation of this curved interface at the plasma membrane of infected cells results in membrane bending and subsequent budding of the virus particles. The structure of the CA hexamer used in our modelling was taken from the intrinsically curved CA lattice within immature virus-like particles [43]. However, the MA domains were arranged to be flat to match the flat model membrane at the beginning of the simulations (Fig. 1D). Interestingly, over the course of the simulations, as the MA-CA linker contracted allowing direct contacts between these two domains, the MA domain spontaneously adapted to the curved CA hexamer (Fig. 2B and S3). Consequently, the curved MA domain induced some degree of curvature in the membrane. This shows how a curved CA-CA interface could affect surrounding Gag domains and the membrane, thereby affecting viral release.

\subsection{Interaction between cleavage site residues and non-cleavage site mutations}

The linker contraction described above resulted in a decrease in solvent accessibility of the cleavage site residues between the MA and CA domains from $\sim 10 \mathrm{~nm}^{2}$ to $\sim 6 \mathrm{~nm}^{2}$ (Fig. 2C and S4). In the first round of proteolysis, Gag is cleaved at the SP1/NC cleavage site to produce MA-CA-SP1 and NC-SP2-P6 intermediates (Fig. S1). In the second round of proteolysis, $\mathrm{P} 6$ is cleaved off the latter at a rate measured in vitro approximately 9-fold slower than the initial cleavage, while MA is cleaved off the former at an estimated 14fold slower rate than the first round of proteolysis [49]. Cleavage at the MA/CA site therefore happens around 1.6 times more slowly than at the SP2/P6 site. Our simulations suggest that this may be caused by the reduction in exposure of the MA/CA cleavage site, whereby at the end of the simulations, the solvent accessible surface area (SASA) of the cleavage site is also approximately 1.6 times smaller than at the beginning of the simulations, due to the contraction of the linker region between MA and CA domains. This suggests that the MA-CA linker dynamics could account for the difference in the rates observed in Gag cleavage, and that the SP2-P6 linker adopts a more extended and less buried conformation.

This linker contraction also led to the cleavage site residues interacting with neighbouring subdomains, predominantly with the C-terminal helix of the MA domain, N-terminal loop of the CA domain, and several inter-helical loops on the CA domain, including the CypA binding loop (Fig. 2D). Two PI-resistant mutations were identified at the interaction sites - G123E and H219Q. To understand how these mutations may affect the cleavage site residues, contact analysis with a cut-off distance of $0.6 \mathrm{~nm}$ was performed between the cleavage site residues and these two specific positions in the WT and mutant proteins, particularly focusing on potential inter- and intra-subunit interactions. Due to their close proximity, we found that the residue at position 123 made contact primarily with the $\mathrm{N}$-terminal portion of the cleavage site from the same Gag subunit (Fig. 3A and 3B). Both WT (glycine) and mutant (glutamate) residues showed a similar percentage of contact over the course of the simulations. "Back-mapping" of CG simulation snapshots to transform them into atomic resolution, performed using a flexible geometric approach [50] (details in Methods section), suggested that a glutamate residue at this position may interact with polar residues on the cleavage site, such as Q130 and Y132 (Fig. 3C). We performed three independent 200 ns 
A

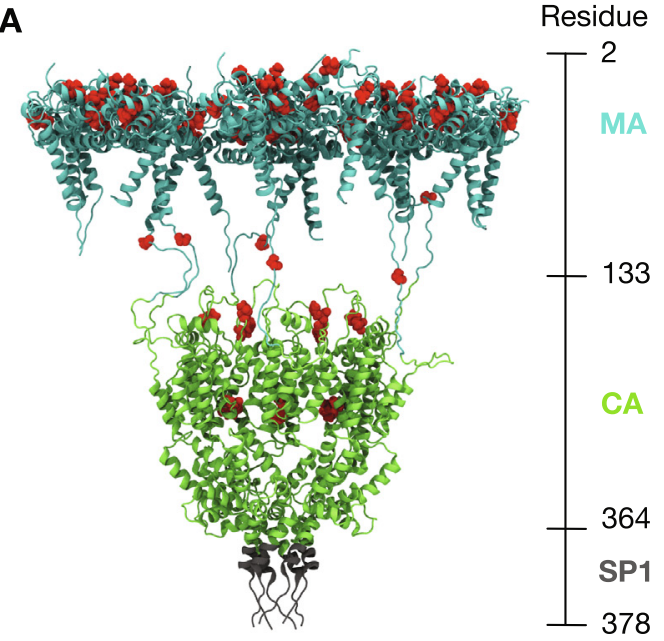

C

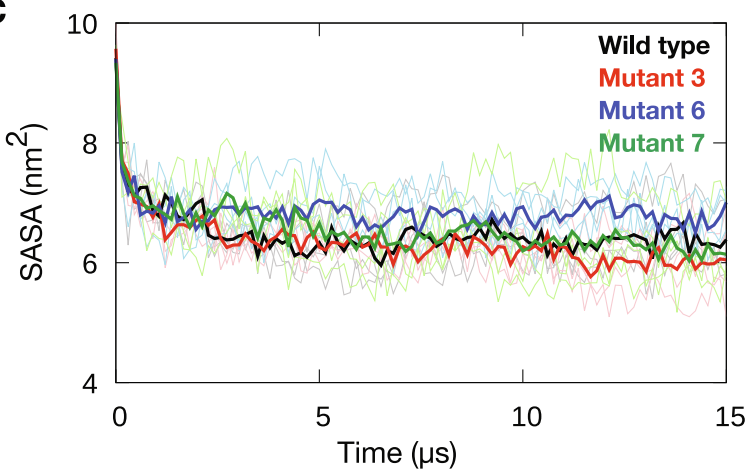

B
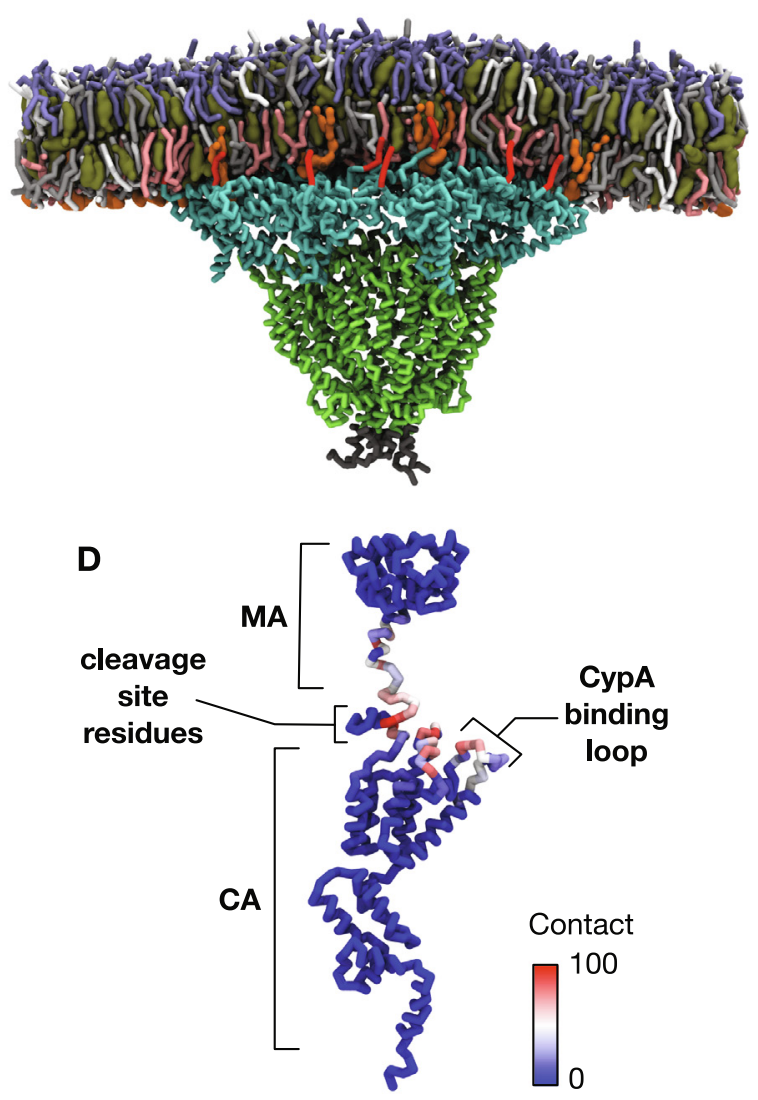

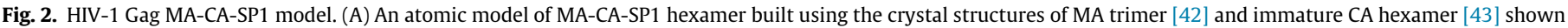

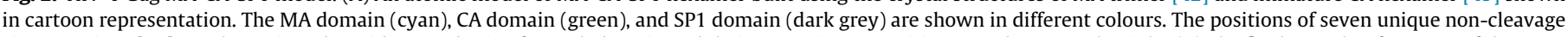

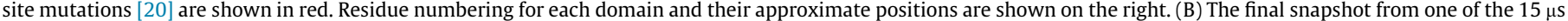

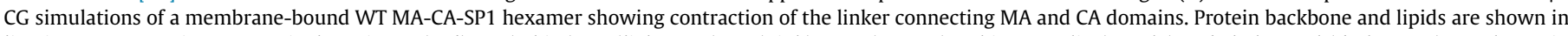

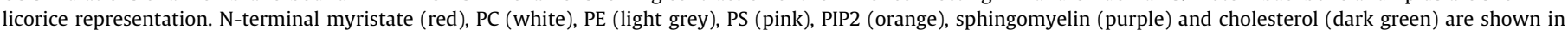

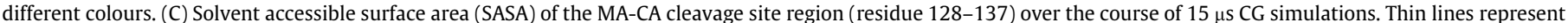

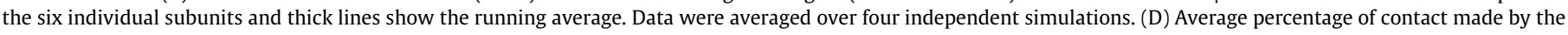

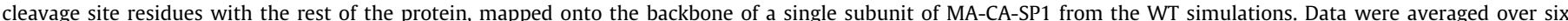

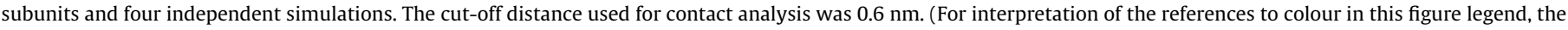
reader is referred to the web version of this article.)

atomistic simulations of a single MA-CA-SP1 subunit to refine the back-mapped structure, and found that the glutamate residue indeed interacted primarily with Y132 (Fig. S5). Additionally, the residue also contacted residues N131 and Q130. In contrast, a glycine residue at this position interacted primarily with V128 and S129. Given the change in the overall size and charge of the residue in the WT and mutants (from small and neutral to large and acidic), the G123E mutation alters the accessibility and electrostatic properties in the vicinity of the cleavage site and would therefore be expected to directly interfere with proteolysis.

While the interactions of G123E with the cleavage site residues occur within the same subunit of the MA-CA-SP1 polyprotein, inter-subunit interactions were observed for H219, whereby this residue contacted the cleavage site of an adjacent subunit (Fig. 3D). The frequency of such interactions, however, was notably lower than that displayed by G123E, and interestingly, the mutation to glutamine resulted in a reduced contact with the cleavage site (Fig. 3E). Back-mapping to atomistic representation suggested potential for interactions with polar residues such as N131 and N137 (Fig. 3F). Atomistic simulations of two adjacent MA-CA-SP1 subunits using the back-mapped structure showed that histidine at this position made intermittent contacts with I134, V135, Q136 and N137, whilst glutamine interacted primarily with Y132
(Fig. S5). Given that the WT histidine may potentially become protonated, it may affect local electrostatic surface properties of the Gag cleavage site to modulate the catalytic efficiency of the protease enzyme.

Overall, our data suggest that even though these mutations occur far from the protease binding site, they can physically interact with the cleavage site residues, either within the same Gag subunit or with a neighbouring subunit, as the linker region between MA and CA domain contracts following the first proteolytic cleavage.

\subsection{Mutations in the MA domain modulate membrane binding}

Four of the mutations in the MA domain map onto the plasma membrane associating surface of our model, and three mutations - E12K, E40K and L75R - involve a switch to basic amino acids. We hypothesized that these mutations may therefore alter key electrostatic interactions with lipids. Indeed, in the three mutant variants, there is a notable increase in positive electrostatic charge on the membrane binding region compared to WT (Fig. S6). In the WT Gag MA domain, the positive charge is concentrated on the peripheral region of the membrane binding surface of the MA trimer. In the mutant variants, in particular mutants 6 and 7, the 
A

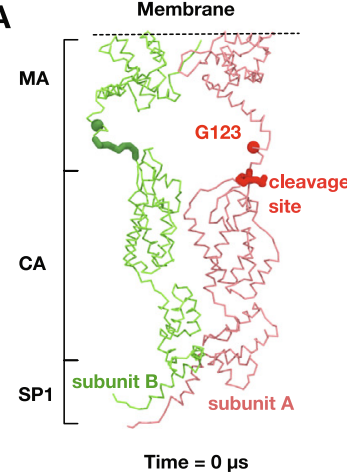

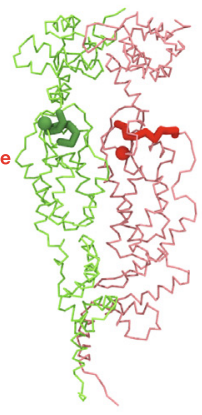

$15 \mu \mathrm{s}$
B

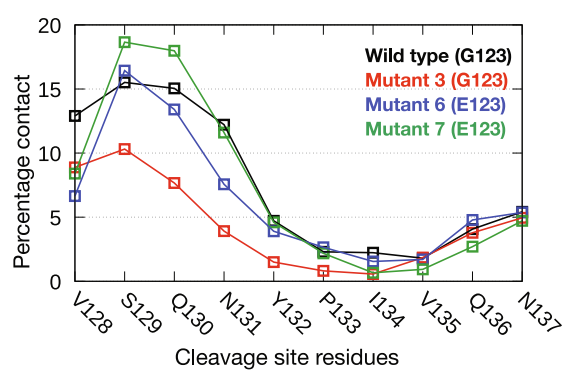

E

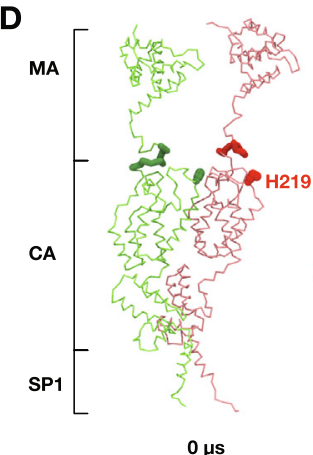

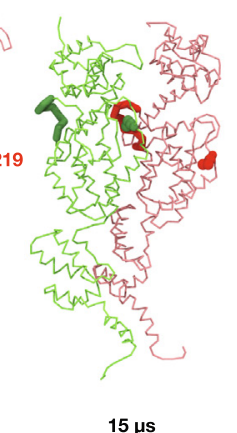

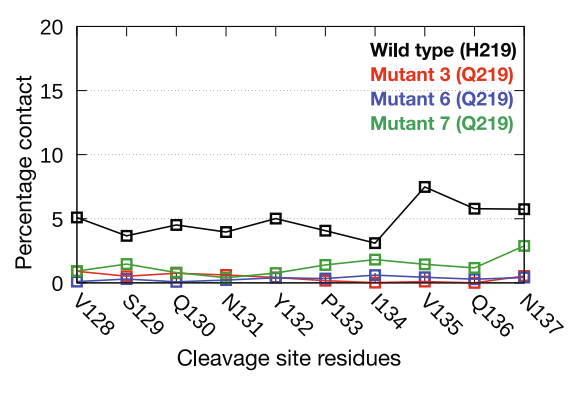

C

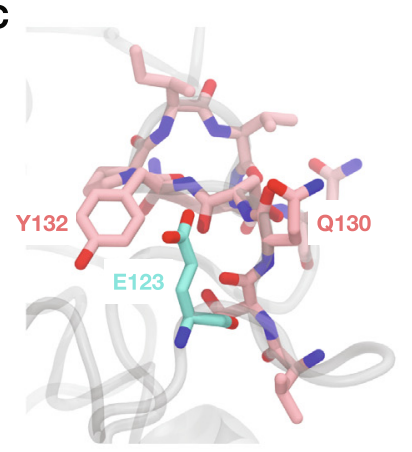

$\mathbf{F}$

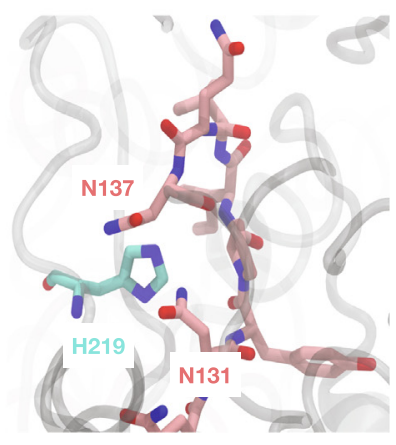

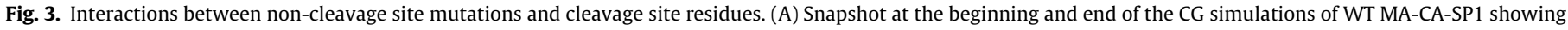

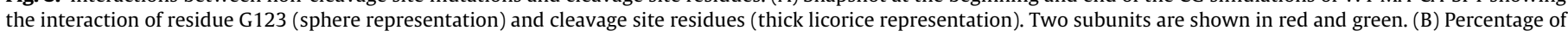

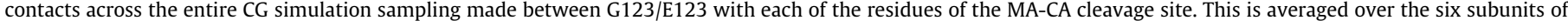

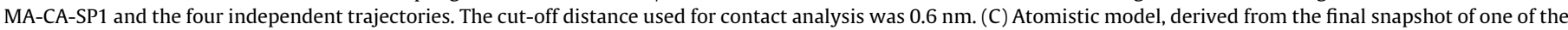

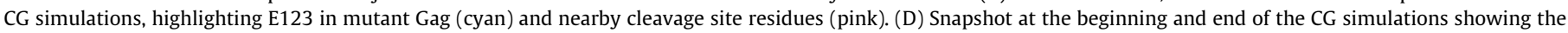

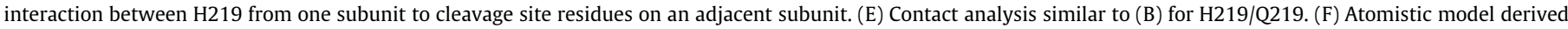

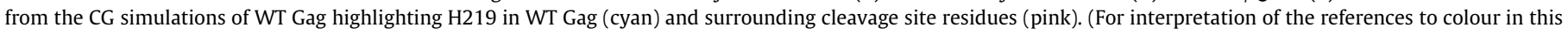
figure legend, the reader is referred to the web version of this article.)

positive charge covers almost the entire membrane binding surface including the central region at the interface of the MA trimer subunits.

Previous biochemical and structural studies showed that PIP2 is instrumental in targeting Gag to the plasma membrane of the host cell, and that it binds directly to the MA domain [51,52]. To understand how the changes in electrostatic surface properties caused by the PI-resistant mutations affect membrane binding, we determined the percentage of PIP2 interacting with MA throughout our CG simulations. This is based on the number of PIP2 molecules found within $0.6 \mathrm{~nm}$ of MA normalized by the total number of PIP2 molecules within the membrane. Over the course of the $15 \mu$ s simulations, there was a significant enrichment of PIP2 lipids around the MA domain, consistently observed across all trajectories, from around $30 \%$ at the beginning of the simulations to 60 $80 \%$ by the end (Fig. $4 \mathrm{~A}$ ). A number-density map averaged over the last $5 \mu \mathrm{s}$ of the simulations showed a significantly higher density of PIP2 around the edges of MA hexamer-of-trimers as well as at the interface between MA trimers compared to areas further away from the protein (Fig. 4B and S7A). This was due to electrostatic attraction between the anionic PIP2 headgroups and the positively charged membrane-peripheral surface of the MA domain. Interestingly, we did not observe any increment in the percentage of anionic PS lipids despite the higher concentration of PS compared to PIP2 in the membrane (Fig. S7B and S7C). This may be due to the higher negative charge on the headgroups of PIP2 lipids. PIP2 enrichment around the MA domain is in agreement with previous experimental studies showing that PIP2 is responsible for anchoring HIV-1 Gag to the plasma membrane [53]. While both WT and mutant variants displayed PIP2 enrichment, all three mutants showed a larger degree of accumulation of PIP2 around MA. More importantly, the E40K and L75R mutations, which map around the interface of the MA trimer subunits, formed novel binding sites for PIP2 lipids (Fig. 4C, 4D). Our simulations therefore suggest that these non-cleavage site mutations enhance interactions between the MA domain and PIP2 lipids, and may consequently improve membrane targeting of HIV-1 Gag during viral assembly.

\subsection{Mutations in CypA-binding loop optimize CypA interaction}

During viral maturation, CA molecules undergo a large conformational change to form a conical capsid encapsulating the viral genome. We next sought to elucidate the impact of non-cleavage site mutations on the mature CA using the crystal structure of the mature CA hexamer [40]. We first investigated the interactions between mature CA and Cyclophilin A (СурA). CypA is a host protein that is recruited by HIV-1 Gag into mature virion particles. While the exact role of CypA in HIV-1 replication remains unclear, it is thought that the prolyl isomerase activity destabilises interactions between the subunits of the CA hexamer and therefore promotes viral core disassembly during the early stage of the replication cycle $[26,54,55]$. An optimal population of CypA in the infected target cell has been shown to be critical in modulating HIV-1 infectivity [56]. At a very low CypA concentration, the HIV-1 replication rate is severely reduced as the CA subunits are tightly bound to one another, which hinders virion uncoating. On the other hand, at a very high CypA concentration, interactions between CA subunits are greatly destabilised, resulting in an unstable virion core and thereby delayed virion maturation [57]. CypA binds to CA via an exposed proline-rich unstructured loop 
A

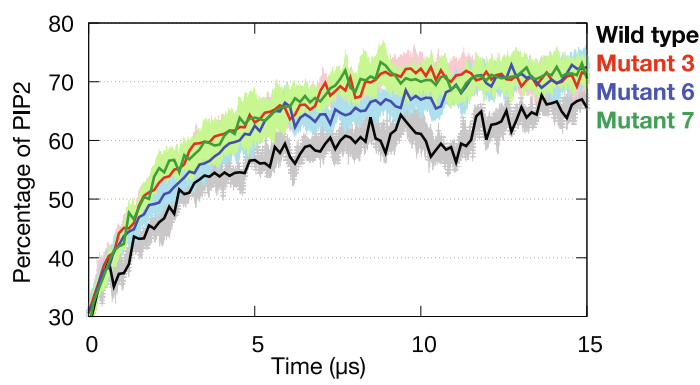

C

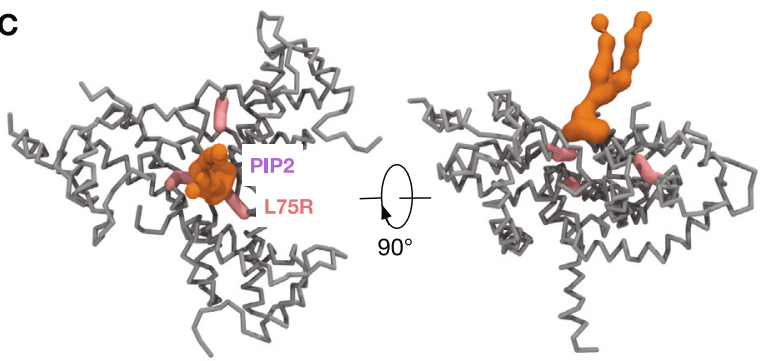

B
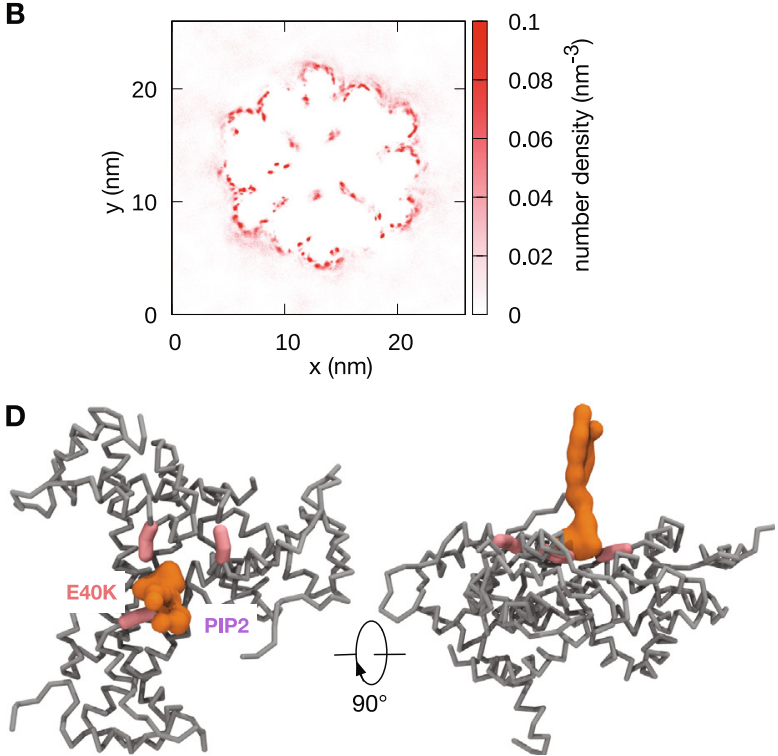

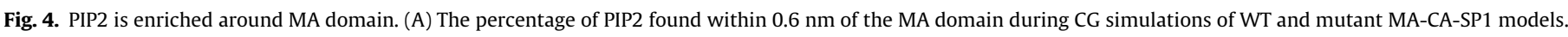

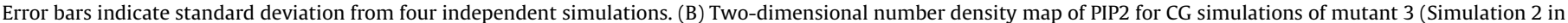

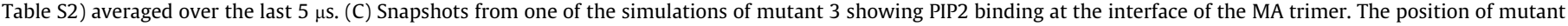

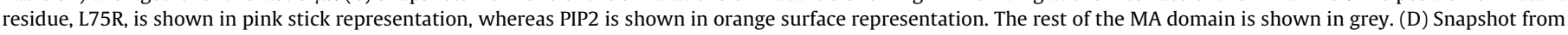

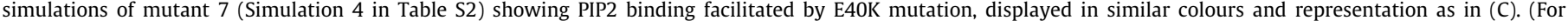
interpretation of the references to colour in this figure legend, the reader is referred to the web version of this article.)

region in the N-terminal domain of the latter [55]. Interestingly, this loop houses $\mathrm{H} 219 \mathrm{Q}$, a mutation found in all seven variants from Gatanaga et al. (Table S1). To investigate the potential role of this mutation vis-à-vis CA-CypA interactions, we performed two independent 500 ns atomistic MD simulations of the WT and H219Q mutant of the mature CA hexamer either in its CypA-free (apo) or CypA-bound state (Simulations 7-12 in Table S2). The predicted pKa values of H219 in all six chains of the hexamer were determined using the PROPKA3 programme [58] and were found to range between 6.3 and 6.5 (Table S3). As these pKa values are close to physiological pH, it is possible that $\mathrm{H} 219$ may exist in either protonated or unprotonated form. As such, the simulations of WT CA were performed with $\mathrm{H} 219$ in both protonation states. IP6 has been shown to be an important co-factor for HIV-1 capsid maturation and assembly [40,59-61]. In the immature Gag hexamer, IP6 interacts with $\mathrm{K} 290$ and $\mathrm{K} 359$, thus facilitating the formation of a six-helix bundle found between the CA and SP1 domains. Proteolytic cleavage between these two domains releases IP6 to subsequently bind to residue R18 in the mature CA, promoting assembly of mature CA hexamers and the lattice. As such, we included the IP6 molecule in all of our atomistic simulations of the mature CA hexamer (see Methods section for further details).

Our apo simulations revealed that the CypA binding loop was the most dynamic region of the entire CA molecules (Fig. 5A). This is consistent with a recent structure of a CA tubular assembly determined by magic-angle spinning NMR by Manman et al. showing that the loop adopts at least four distinct conformations [62]. No noticeable difference was found between the flexibility of this loop in simulations with the neutral H219 versus the H219Q mutant (Fig. 5B). Interestingly, when this histidine residue was protonated, we saw a slight increase in the root mean square fluctuation (RMSF) values of the loop, especially around G221-P222, which is the putative substrate for the CypA rotamase activity $[63,64]$. Cluster analysis performed on structures extracted from the simulations revealed that the СурА binding loop containing the protonated $\mathrm{H} 219$ was the most heterogenous with the largest distribution of root mean square deviation (RMSD) values, the highest number of distinct clusters, and the fewest structures grouped into the top three clusters (Fig. S8). An increased association was observed between protonated H219 and E230, found on the opposite side of the loop (Fig. 5C). Similarly, the central structure of the top cluster from protonated H219 simulations showed formation of a salt bridge between the protonated histidine and E230 (Fig. 5D), which was absent from the top three clusters extracted from simulations with neutral H219 and H219Q mutant, as well as the ensemble of wild type CA structures solved by Manman et al. [62] (Fig. S8). However, this salt bridge was intermittent (Fig. 5E), such that the CypA-binding loop may adopt alternative conformational states when $\mathrm{H} 219$ is protonated. This means that under low $\mathrm{pH}$ conditions, the transition between these two states increases the overall flexibility of the loop, which consequently may make it more difficult for CypA to bind. Mutation of H219 to glutamine would therefore abolish the ability of the loop to adopt these two conformational states, reducing the loop flexibility, and facilitate initial CypA binding. This would be advantageous inside the endosome which is associated with a lower local $\mathrm{pH}$, to ensure sufficient CypA binding for core disassembly [65].

Since the interactions between H219 and CypA form a part of the binding interface ${ }^{55}$, this raises the question of whether a mutation to glutamine would undermine the strength of binding. To investigate the potential effects of the $\mathrm{H} 219 \mathrm{Q}$ mutation upon CypA binding affinity, we performed two independent 500 ns atomistic MD simulations of a mature CA hexamer with each of the subunits bound to a CypA molecule (Fig. 6A). While the average buried surface area between CA and CypA in the WT and mutant CA systems were comparable throughout the simulations, we observed transient detachment of CypA molecules in two of the CA subunits from the $\mathrm{H} 219 \mathrm{Q}$ variant (Fig. 6B). Based on the crystal structure [55] H219 forms hydrogen bonds with residues in the CypA active site, including N71. Our simulations revealed that this histidine residue also interacts with other polar residues such as T73, N102, and Q111 on CypA. In the H219Q mutant, the contacts made by the glutamine residue followed a largely similar pattern, although the frequency of interactions was noticeably lower com- 

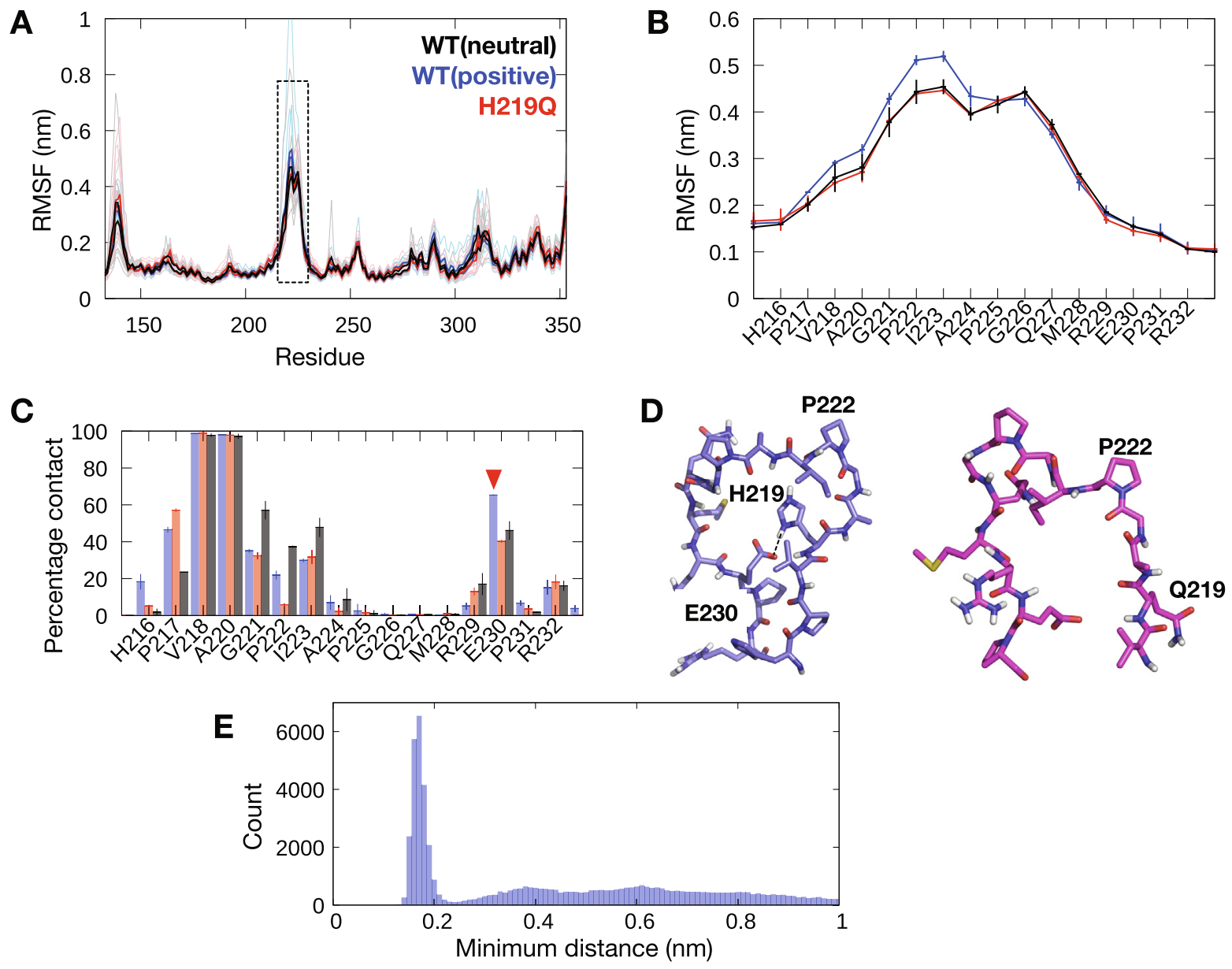

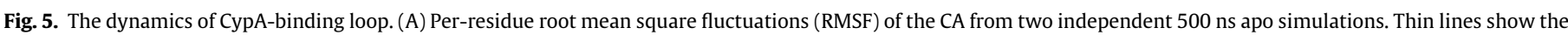

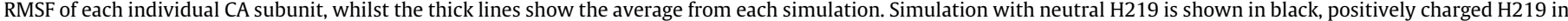

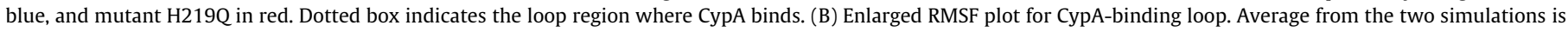

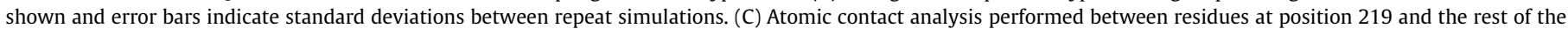

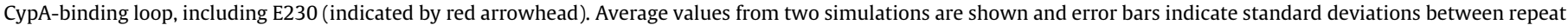

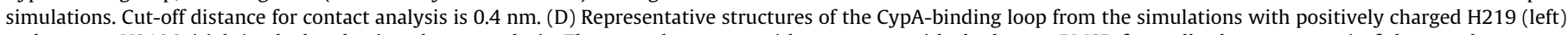

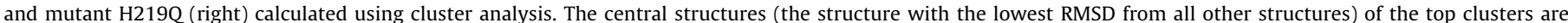

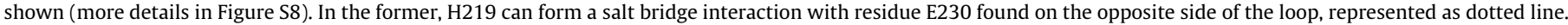

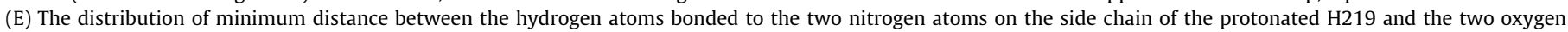

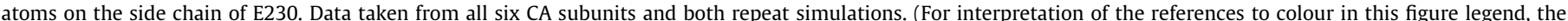
reader is referred to the web version of this article.)

pared to both neutral and protonated histidine (Fig. 6C). Both of these analyses of CypA-bound CA simulations suggest that the H219Q mutation may potentially weaken CypA binding to the CA domain. This mutation is therefore likely beneficial in a CypArich environment whereby a high population of CypA in target cells may destabilise the viral core. Taken together, the H219Q mutation on the CypA-binding loop may play a crucial role in fine-tuning CypA interactions, especially in low endosomal $\mathrm{pH}$ and СypAenriched infected target cells.

It is worth noting that cryo-EM studies of the large HIV-1 capsid lattice showed that a single CypA molecule could simultaneously bind two CA hexamers (Fig. S9) [38,66]. To one hexamer, CypA binds to one CA subunit via canonical interactions with the CypA-binding loop discussed in this study. To the other neighbouring hexamer, CypA binds to two adjacent CA subunits via noncanonical interactions, facilitated by residues $254-257$ as well as 227-229. We compared the RMSF values of these residues between our three sets of apo simulations involving neutral $\mathrm{H} 219$, protonated $\mathrm{H} 219$ and mutated $\mathrm{H} 219 \mathrm{Q}$ variants (Simulations 7-9 in Table S2). There was no significant difference between the RMSF values for these residues, suggesting that the protonation and mutation of $\mathrm{H} 219$ would not affect the dynamics of residues involved in non-canonical binding of CypA. Interestingly, the cryo-EM structures show that these two non-canonical binding interfaces have a much smaller interaction area compared to the canonical interface, suggesting a weaker interaction. This is supported by the lower pairwise interaction energies of the noncanonical interface from previous MD simulations [38]. Collectively, these non-canonical CypA-CA interfaces act as a secondary binding site, once CypA binds to the canonical CypA-binding loop. While we focused on the primary CypA binding site, since mutation and protonation of residue $\mathrm{H} 219$ does not affect the dynamics of the residues at the non-canonical interfaces, it is conceivable that CypA binding at these secondary sites may not be regulated by the $\mathrm{H} 219 \mathrm{Q}$ mutation.

\subsection{Mutation in the CA hexameric interface stabilizes oligomerisation}

Finally, another non-cleavage site mutation in the CA domain occurs at position 199, in which a glutamine residue is mutated to a histidine [20]. In immature Gag, this residue is located inside the CA domain pointing towards the internal cavity. Our CG 
A

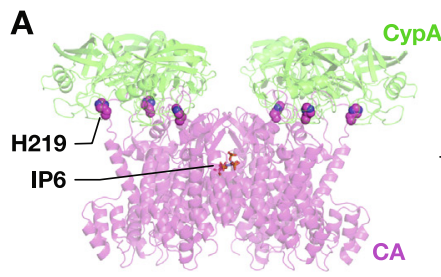

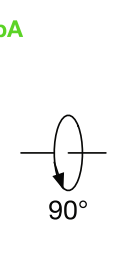

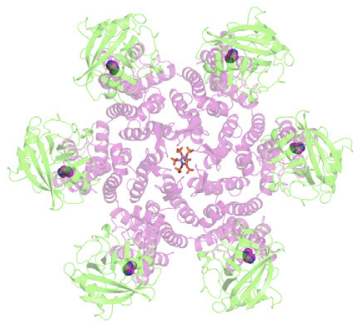

C $\quad \mathrm{H} 219$ (neutral)

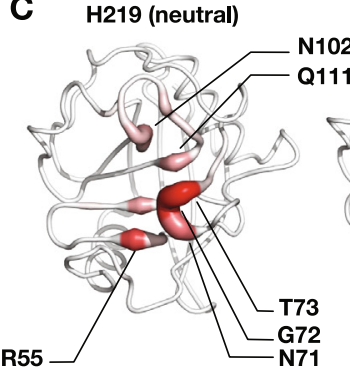

H219 (positive)

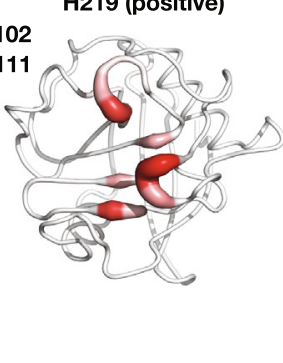

B

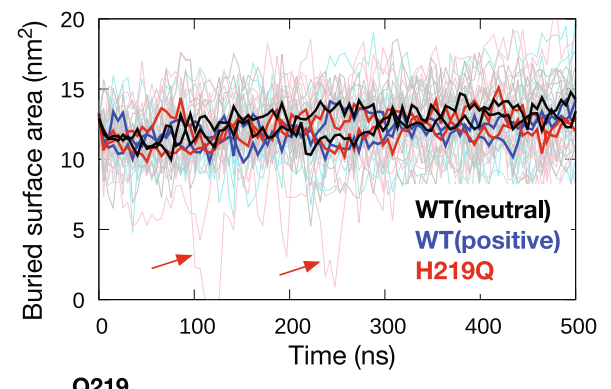

Q219

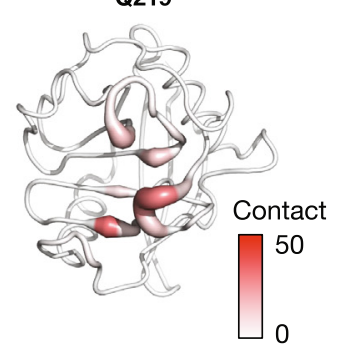

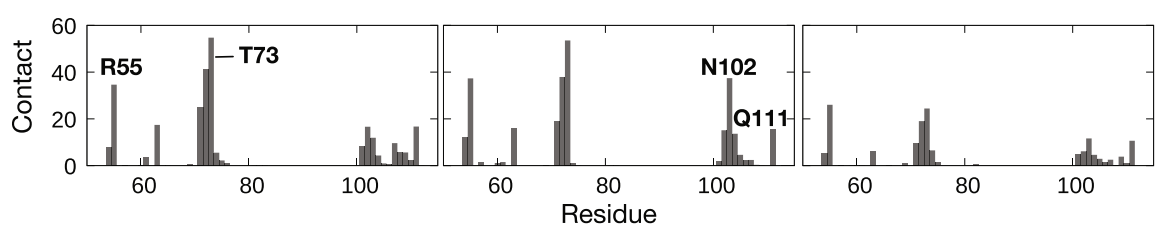

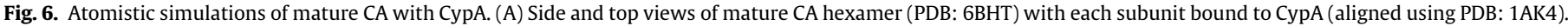

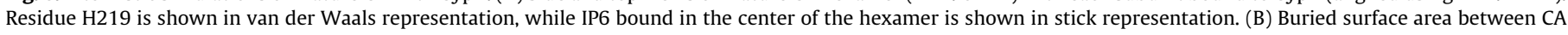

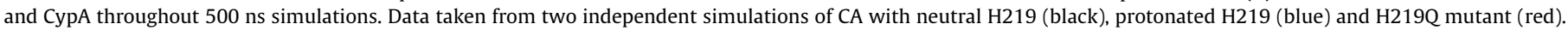

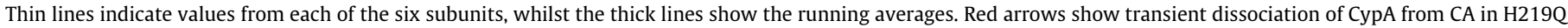

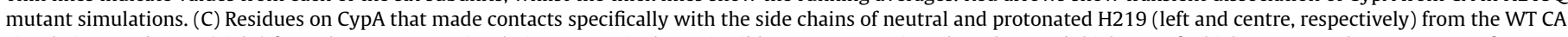

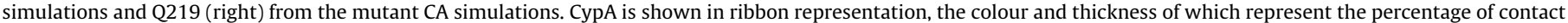

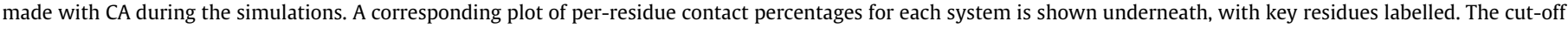

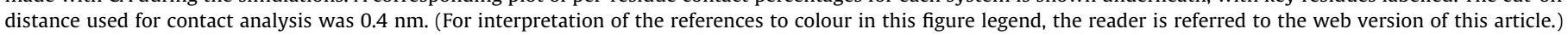

simulations show that this residue did not form any major interactions with other CA residues as it was fully exposed to the solvent within the cavity of the CA. However, as CA undergoes large conformational changes during maturation, the Q199 residue forms a part of the hexameric interface of a mature $C A$ domain (Fig. 7A). This residue bridges the $\mathrm{N}$-terminal domain of one $\mathrm{CA}$ subunit and the C-terminal domain of an adjacent subunit via polar interactions with residues such as Y301, K314 and Q311. We hypothesized that a mutation to histidine may alter the interactions between the two CA subdomains and therefore affect oligomerisation.

To test this hypothesis, we performed two repeat 500 ns atomistic simulations of mature CA for the WT and Q199H variants, the latter in either protonated or unprotonated states (Simulations 7, 13 and 14 in Table S2) (Fig. 7B). We found that both neutral and charged histidine retained all major interactions as observed for glutamine in the WT protein; for example, the two most prevalent interacting partners from the neighbouring subunit were Y301 and L343, via polar and van der Waals interactions, respectively. Similar to the WT simulations, these residues were found to interact with H199 throughout most of the trajectories. However, the mutant simulations also showed additional contacts with some residues compared to WT. Thus, neutral H199 made significantly more frequent interactions with K314 and V313, while protonated H199 could form a salt bridge with E319. Protonation of H199 also modulates some interactions at the hexameric interface; for example, contact with R305 is noticeably reduced due to electrostatic repulsion, whilst intermittent contacts were observed with Q311 and E312, which explains the large standard deviations between the two repeat simulations. Pair interaction energies of both neutral and protonated $\mathrm{H} 199$ with surrounding residues from the neighbouring subunit are significantly more negative than that of Q199 (Fig. 7C), indicating stronger interactions made by this histidine residue at the CA hexameric interface compared to the wild type glutamine. Our simulations therefore suggest that a histidine residue at position 199 potentially strengthens the binding interface between CA subunits to enhance the stability of the hexameric complex.

\section{Discussion}

Given the possible benefits of drugging Gag together with the protease, we set out to study cleaved Gag structures using multiscale modelling and simulations of the HIV-1 Gag protein to understand the effect of clinically relevant mutations that occur outside of the cleavage sites. We built a model of the MA-CA-SP1 polyprotein complex in its multimeric state, which constitutes the largest cleavage product of the immature Gag protein. Our simulations of the membrane-bound complex suggest that the long linker connecting the MA and CA domains is flexible and may contract, enabling interactions between protease cleavage site residues and the C-terminal region of the MA domain, as well as several regions on the CA domain including the CypA binding loop. Two clinical mutations, G123E and H219Q, are found in these regions and alter the accessibility and electrostatic properties of the protease binding site. Mutations in the MA domain are concentrated on the predicted membrane binding surface and at least three of them - E12K, E40K and L75R - render the surface more positively charged compared to wild type Gag. Our CG simulations of these mutant variants show more pronounced interactions with PIP2 

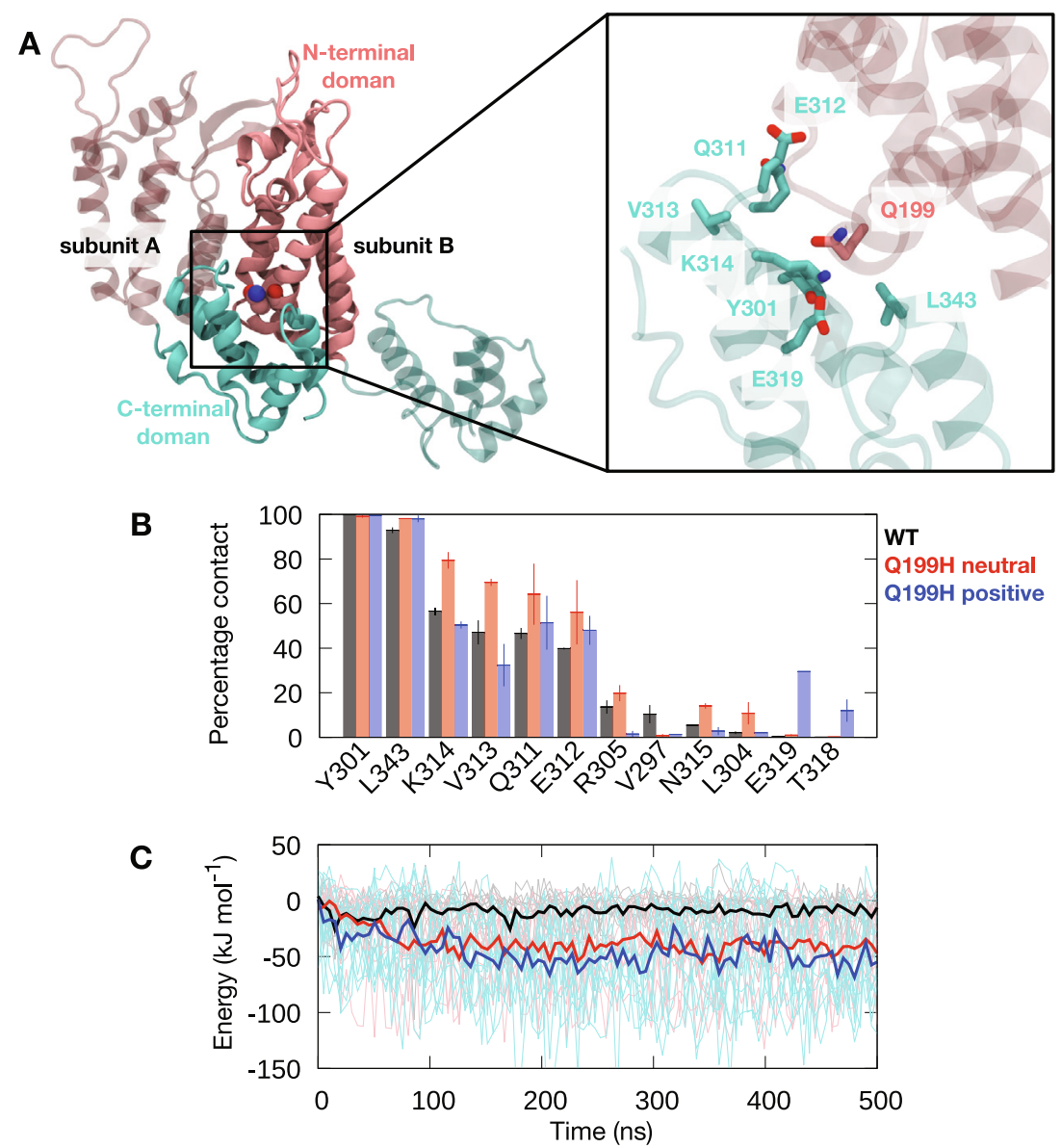

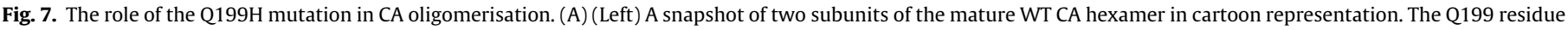

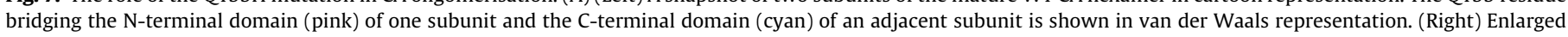

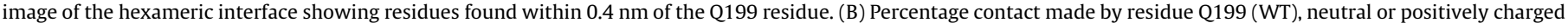

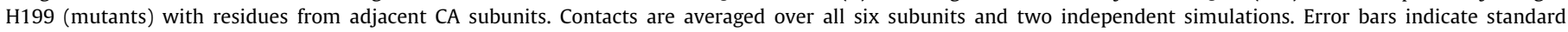

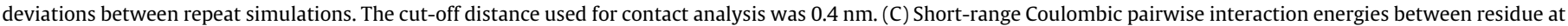

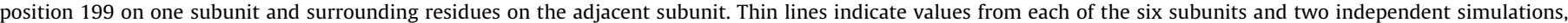

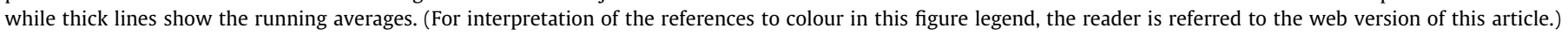

lipids, leading to the hypothesis that these mutations improve Gag targeting and anchoring to the plasma membrane during viral assembly. Atomic-resolution simulations of the mature Gag CA domain showed that the $\mathrm{H} 219 \mathrm{Q}$ mutation may play a role in optimizing CypA interactions by controlling the conformational flexibility of the СypA binding loop and fine-tuning the CypA binding affinity. We also found that a mutation in the CA domain, Q199H, likely enhances oligomerisation by providing more stabilising interactions between adjacent subunits.

In the field of HIV and other related retroviruses, multiscale modelling and simulations have been utilised to study CA in different maturation states, providing important insights into their structural dynamics and interactions with drugs and host cell proteins [38,67-69]. For example, landmark microsecond-timescale atomic simulations of the entire HIV-1 capsid revealed important physicochemical properties, such as its electrostatics, dynamics, and water/ion permeability, as well as pointing towards longrange allosteric motions in the shell [35]. Simulations of the monomeric MA domain shed light on the molecular mechanism underlying membrane anchoring by the myristoyl group and PIP2 lipids [41]. Our study reports the first complete oligomeric model of the proteolytic product of HIV-1 Gag polyprotein, allowing the investigation of interactions between residues on the protease binding site and distant residues on the MA and CA domains, especially those from different subunits. Such inter-subunit "crosstalk" would be difficult to identify using individual crystal structures of mature Gag domains or a monomeric Gag model. Understanding structural changes that occur upon proteolysis could provide important clues on HIV-1 protease activity. For example, the MACA linker contraction observed in our simulations resulted in a significant decrease in solvent accessibility of the protease binding site, which helps to explain the lower cleavage rate of the MA-CA domain compared to the SP2-P6 domain [49]. From the perspective of anti-HIV drug development, targeting Gag proteolytic products in addition to the mature form, by virtue of identifying intermediate structural models, may allow for the discovery of Gag inhibitors that can inhibit multiple stages of maturation [14].

Since accumulation of deleterious mutations could negatively impact virion survival, Gag polyprotein and protease cocompensate to outcompete protease inhibitors and restore viral fitness $[14,15,70]$. While HIV-1 protease acquires drug resistant mutations to evade PI activity, its ability to bind and subsequently cleave the Gag protein is impaired. Thus, Gag gains additional mutations to compensate for fitness defects to recover protease function. Structural studies show that associated mutations on cleavage sites do not restore interactions lost due to mutations on the protease, but rather establish novel interactions surrounding the site of protease mutations as well as induce conformational changes to drive better binding [18]. Apart from cleavage site mutations, mutations in more distant regions have been shown 
to contribute towards recovery from the reduced viral fitness caused by protease mutations [19,20]. Our study shows that these non-cleavage site mutations have far reaching implications outside of Gag proteolysis, from improving membrane binding to finetuning СурA interactions and stabilising the CA hexamer. A recent single genome sequencing study revealed that, indeed, most Gagprotease correlated mutations occur outside of the Gag cleavage site with the viral MA and CA domains representing the largest subsets of the mutations [70]. Interestingly, a high concentration of compensatory mutations was uncovered within the globular domain of MA (which binds to the plasma membrane) and the CypA binding loop of CA, further corroborating our simulation results. Mutations and deletions of residues on the C-terminal region of the MA domain, which have direct contact with cleavage site residues based on our simulations, have also been recently shown to confer resistance to PI lopinavir [71]. Our results also highlight that the residues on the CypA binding loop can interact directly with MA-CA cleavage site residues, thus potentially influencing their local electrostatics and surface accessibility, although determining the exact interactions made between these residues and subsequent molecular effects would require further studies. Additionally, PS lipids are known to be exposed on the outer leaflet of HIV-1 membrane and may facilitate HIV-1 entry into the host cells $[72,73]$ and it is possible that the highly basic surface generated by the non-cleavage site mutations could attract anionic PS lipids, similarly to the PIP2 enrichment around the MA domain observed here. While our study assumes that these non-cleavage site mutations do not have any large impact on the overall structure of Gag, it is possible that these point mutations could result in local structural changes. Further structural and computational studies would thus be of interest to directly elucidate the impact of these mutations on Gag structure. It is worth noting that compensatory non-cleavage site mutations also occur in the NC and P6 domains. Gatanaga et al. discovered five such mutations with clinical importance [20] and many clinical drug resistant mutations can occur within the first replication cycle [7]. While modelling these domains is beyond the scope of the current study, it is certainly worth exploring in the future, specifically regarding how these mutations affect interactions with the viral RNA genome. Overall, our data have revealed new insights into the role of Gag non-cleavage site mutations on HIV-1 viral fitness. Given that HIV-1 is one of the fastest mutating RNA viruses, an in-depth comprehension of emerging mutations will aid in the general understanding of viral drug resistance, as well as the emergence of novel viruses that are able to cross species during zoonotic infections.

\section{Materials and methods}

\subsection{Model building and system setup}

The model of HIV-1 Gag MA-CA-SP1 was built using two structural templates: MA trimers (PDB: 1HIW) [42] and the immature CA hexamer (PDB: 5L93) [43]. To the best of our knowledge, the former represents the only available crystal structure of the MA protein in its trimeric form, whilst the latter represents the highest resolution structure of a complete immature CA hexamer. Six copies of MA trimers were arranged based on EM images showing that they organize as hexamer-of-trimers on a model membrane bilayer [44]. The CA hexamer was placed such that the N-termini of its subunits align with the C-termini of the central subunits of the MA trimers. The MA and CA domains are connected by a flexible, disordered linker as shown by solution NMR studies $[45,46]$. Modeller version 9.21 [74] was used to build the loops connecting the MA trimers and CA hexamer (Fig. S1) using the discreet optimized protein energy (DOPE)-based loop modelling protocol, and the model with the lowest DOPE score was chosen [75]. Stereochemical assessment using Ramachandran analysis [76] showed only one outlier residue, confirming that the model was structurally reasonable. The atomistic MA-CA-SP1 structure was subsequently converted to CG representation using the MARTINI 2.2 force field [77] and the elastic network model, ElNeDyn [78] was imposed to maintain the integrity of the secondary and tertiary structure. This CG model was validated by comparing the MA-CA domain dynamics to all-atom simulations of a monomeric MACA-SP1 protein (more details below). The $\mathrm{N}$-terminal glycine residue on each MA subunit was myristoylated based on parameters from Charlier et al. [41] Three mutant variants were generated using PyMOL [79] based on non-cleavage site mutations published by Gatanaga et al. [20] The wild-type residues were substituted with the mutant versions using the PyMOL mutagenesis tool, assuming these point mutations would not result in any large structural deviations from wild-type Gag.

A $30 \times 30 \mathrm{~nm}^{2}$ patch representing the HIV-1 membrane model was built using the CHARMM-GUI Martini Maker Bilayer Builder $[80,81]$ and the lipid composition was based on a previous HIV-1 lipidomic study [47]. This membrane was asymmetric: the upper leaflet was made of $10 \%$ phosphatidyl choline (PC), $10 \%$ phosphatidyl ethanol amine (PE), 30\% sphingomyelin, and 50\% cholesterol, whereas the lower leaflet was made of $10 \%$ PC, 20\% PE, $15 \%$ phosphatidyl serine (PS), 5\% phosphatidylinositol-4,5-bispho sphate (PIP2), and 50\% cholesterol. The HIV-1 membrane is enriched in saturated lipid species especially for PC; we therefore modelled the lipid tails of PC as 1,2-dipalmitoyl (DP), whereas the rest of the lipids were modelled with 1-palmitoyl-2-oleoyl (PO) lipid tails. Following minimization and equilibration procedures according to the CHARMM-GUI, the model membrane was further simulated for $1 \mu$ s to allow better mixing of the lipid components prior to simulations in the presence of protein.

\subsection{Molecular dynamics simulations}

The HIV-1 Gag MA-CA-SP1 CG model was placed underneath the membrane such that the myristoylated N-termini were inserted into lower leaflet of the membrane. Energy minimization was then performed using the steepest descent method to remove any overlapping beads. The system was solvated with standard MARTINI water molecules and neutralized with $0.15 \mathrm{M} \mathrm{NaCl}$ ions. Further energy minimization was performed. The system was then equilibrated for $10 \mathrm{~ns}$ whereby positional restraints with a force constant of $1000 \mathrm{~kJ} \mathrm{~mol}^{-1} \mathrm{~nm}^{-2}$ were imposed on all of the backbone atoms of the protein. Temperature coupling using the Vrescale thermostat with a time constant of 1 ps [82] was applied to maintain the temperature at $310 \mathrm{~K}$. Semi-isotropic pressure coupling using the Berendsen barostat with a time constant of 5 ps was utilized to maintain the pressure of the system at $1 \mathrm{~atm}$. Electrostatics were calculated using the reaction field method. The der Waals interactions were computed using a potential shift Verlet scheme. The short-range cut-offs for both of these were set to $1.1 \mathrm{~nm}$. After the equilibration simulations, four independent production runs of $15 \mu$ s were performed using different distributions of initial velocities. The same protocols used for the equilibration simulation were used for the production runs in the absence of position restraints, except for the pressure coupling in which the Parrinello-Rahman barostat was used with a coupling constant of 12.0 ps [83].

Atomistic MD simulations were performed to understand the role of the H219Q mutations on CypA binding and Q199H on CA oligomerisation. The structure of the mature HIV-1 CA hexamer in complex with inositol IP6 (PDB: 6BHT) [40] was aligned to that of the CA N-terminal domain bound to CypA (PDB: 1AK4) [55]. This 
alignment generated a structure of the CA hexamer bound to six copies of CypA (Fig. 6A). Mutations in the CA were performed using PyMOL. The PROPKA3 programme [58] was used to calculate the pKa of H219 (Table S3). The proteins were parameterized using the CHARMM36 force field [84] whereas the CHARMM-GUI Ligand Reader \& Modeller [85] was used to generate the parameters for the IP6 molecule. The complex was solvated with TIP3P water molecules and $0.15 \mathrm{M} \mathrm{NaCl}$ was added to neutralize the system. The steepest descent minimization protocol was used to remove overlapping atoms. A short 100 ps equilibration simulation with position restraints (force constant of $1000 \mathrm{~kJ} \mathrm{~mol}^{-1} \mathrm{~nm}^{-2}$ ) applied to all heavy atoms of the protein was conducted. The temperature of the system was maintained at $310 \mathrm{~K}$ using the Nosé-Hoover thermostat with a time constant of 1.0 ps $[86,87]$. The pressure was kept at $1 \mathrm{~atm}$ using an isotropic coupling to the ParrinelloRahman barostat with a time constant of 5.0 ps [83]. Electrostatic interactions were measured using the smooth particle mesh Ewald (PME) method with a real-space cut off of $1.2 \mathrm{~nm}$ [88]. The van der Waals interactions were calculated using the force switch smoothing function applied between 1.0 and $1.2 \mathrm{~nm}$, and truncated at $1.2 \mathrm{~nm}$. An integration time step of $2 \mathrm{fs}$ was used with all covalent bonds involving hydrogens constrained using the LINCS algorithm [89]. After equilibration, the positional restraints were removed and two independent 500 ns production runs were conducted using the same setup for each of the WT and mutant variants starting with different initial velocities. To assess whether the systems were properly equilibrated, we calculated the RMSD of the C $\alpha$ atoms of CA (Simulation 7-14 in Table S2) and CypA (Simulation 10-12 in Table S2) for all six chains of the hexamer (Fig. S10). We found that in most cases the RMSD reached a plateau after around $100 \mathrm{~ns}$.

Additional atomistic and CG simulations were performed to verify the conformation of the MA-CA linker. A single subunit of the HIV-1 Gag MA-CA-SP1 model was placed in solution with $0.15 \mathrm{M} \mathrm{NaCl}$ ions. Positional restraints with a force constant of $1000 \mathrm{~kJ} \mathrm{~mol}^{-1} \mathrm{~nm}^{-2}$ were applied to the backbone atoms of membrane binding residues (residue 2-53 and 72-90) to mimic membrane binding. Four independent 500 ns simulations were performed for both atomistic and CG systems using the same protocols described above.

To refine interactions between the linker region and mutant residues observed in the CG simulations of the MA-CA-SP1 hexamer, we converted the structure of the hexamer from the final frame of one of the simulations to atomistic representation using the CHARMM-GUI All-Atom Converter. The back-mapping protocol was performed using a flexible geometric approach via the backward.py program [50]. In this approach, the atomic particles are mapped to the weighted average of the CG beads, followed by geometrical corrections whereby the atomic particles are repositioned (when necessary) to reconstruct chiral centres and double bond configurations. Force field-based relaxation consisting of a series of energy minimizations and position restrained MD simulations was then conducted. A single subunit of the MA-CA-SP1 was subsequently used to refine interactions with the G123E mutant, whereas two adjacent subunits were used for interactions with the H219Q mutant. The protein was inserted into a box of water with $0.15 \mathrm{M} \mathrm{NaCl}$ ions. Positional restraints with a force constant of $1000 \mathrm{~kJ} \mathrm{~mol}^{-1} \mathrm{~nm}^{-2}$ were imposed on the backbone atoms of residues outside of the MA-CA linker and CypA binding loop (residue 2-117, 147-215 and 233-376). For each system, three independent 200 ns simulations were performed using the parameters described above.

All simulations were performed using GROMACS 2018 [90]. The list of simulations performed is provided in Table S2. Analysis of the electrostatic surface charge of the MA domain was performed in PyMOL using the APBS plugin [91]. Cluster analysis of the СypA binding loop (residue 216-232) was performed on structures generated from simulation trajectories using the GROMACS gmx cluster tool. The clustering algorithm described by Daura et al. [92] was applied using a cutoff of $0.3 \mathrm{~nm}$.

\section{CRediT authorship contribution statement}

Firdaus Samsudin: Investigation, Formal analysis, Visualization, Writing - original draft, Writing - review \& editing. Samuel Ken-En Gan: Conceptualization, Project administration, Supervision, Writing - original draft, Writing - review \& editing. Peter J. Bond: Conceptualization, Project administration, Supervision, Writing - original draft, Writing - review \& editing.

\section{Declaration of Competing Interest}

The authors declare that they have no known competing financial interests or personal relationships that could have appeared to influence the work reported in this paper.

\section{Acknowledgments}

This work was supported by BII and EDDC of A*STAR. Simulations were performed on the petascale computer cluster ASPIRE1 at the National Supercomputing Centre of Singapore (NSCC) and the $\mathrm{A}^{*}$ STAR Computational Resource Centre ( $\left.\mathrm{A}^{*} \mathrm{CRC}\right)$.

\section{Appendix A. Supplementary data}

Supplementary data to this article can be found online at https://doi.org/10.1016/j.csbj.2020.12.022.

\section{References}

[1] Yeo JY, Goh GR, Tran-To Su C, Ken-En Gan S. The determination of HIV-1 RT mutation rate, its possible allosteric effects, and its implications on drug resistance. Viruses 2020;12:1-22.

[2] Duffy S, Shackelton LA, Holmes EC. Rates of evolutionary change in viruses: patterns and determinants. Nat Rev Genet 2008;9:267-76.

[3] Li W et al. Bats are natural reservoirs of SARS-like coronaviruses. Science (80-.) 2005;310:676-9.

[4] Kuiken T et al. Host species barriers to influenza virus infections. Science (80-.) 2006;312:394-7.

[5] Wu F et al. A new coronavirus associated with human respiratory disease in China. Nature 2020;579(7798):265-9. https://doi.org/10.1038/s41586-0202008-3.

[6] Zhou P et al. A pneumonia outbreak associated with a new coronavirus of probable bat origin. Nature 2020;2019.

[7] J. Yi Yeo, P. Yap, G. Goh, D.W. Koh, S.K. Gan, HIV-1 mutations in HIV-1 Gag , protease, RT p66 and when they appear : Insights from an in vitro BSL2 assay on mutation rates and types . bioRxiv 1-26 (2020).

[8] Perelson Alan S. Modelling viral and immune system dynamics. Nat Rev Immunol 2002;2(1):28-36.

[9] Collier DA, Monit C, Gupta RK. The Impact of HIV-1 Drug Escape on the Global Treatment Landscape. Cell Host Microbe 2019;26(1):48-60.

[10] Gregson J et al. Global epidemiology of drug resistance after failure of WHO recommended first-line regimens for adult HIV-1 infection: A multicentre retrospective cohort study. Lancet Infect Dis 2016;16:565-75.

[11] Su CTT, Ling WL, Lua WH, Haw YX, Gan SKE. Structural analyses of 2015updated drug-resistant mutations in hiv-1 protease: An implication of protease inhibitor cross-resistance. BMC Bioinf. 2016;17(S19). https://doi. org/10.1186/s12859-016-1372-3.

[12] Ajose O, Mookerjee S, Mills EJ, Boulle A, Ford N. Treatment outcomes of patients on second-line antiretroviral therapy in resource-limited settings: a systematic review and meta-analysis. Aids 2012;26(8):929-38.

[13] Collier D et al. Virological outcomes of second-line protease inhibitor-based treatment for human immunodeficiency virus type 1 in a high-prevalence Rural South African setting: A competing-risks prospective cohort analysis. Clin Infect Dis 2017;64:1006-16.

[14] Su CT-T, Koh DW-S, Gan SK-E. Reviewing HIV-1 Gag mutations in protease inhibitors resistance: insights for possible novel gag inhibitor designs. Molecules 2019;24(18):3243. https://doi.org/10.3390/molecules24183243.

[15] Clavel F, Mammano F. Role of gag in HIV resistance to protease inhibitors. Viruses 2010;2(7):1411-26. 
[16] Sutherland KA et al. Gag-protease sequence evolution following protease inhibitor monotherapy treatment failure in HIV-1 viruses circulating in East Africa. AIDS Res Hum Retroviruses 2015;31(10):1032-7.

[17] Gupta RK, Kohli A, Mccormick AL, Towers GJ. Full length HIV-1 Gag determines protease inhibitor susceptibility within in vitro assays. Aids 2010;24 (11):1651-5.

[18] Özen A, Lin KH, Yilmaz NK, Schiffer CA. Structural basis and distal effects of Gag substrate coevolution in drug resistance to HIV-1 protease. PNAS 2014;111:15993-8.

[19] Myint L et al. Gag non-cleavage site mutations contribute to full recovery of viral fitness in protease inhibitor-resistant human immunodeficiency virus type 1. Antimicrob Agents Chemother 2004;48:444-52.

[20] Gatanaga $\mathrm{H}$ et al. Amino acid substitutions in Gag protein at non-cleavage sites are indispensable for the development of a high multitude of HIV-1 resistance against protease inhibitors. J Biol Chem 2002;277:5952-61.

[21] Sutherland KA et al. Evidence for reduced drug susceptibility without emergence of major protease mutations following protease inhibitor monotherapy failure in the SARA trial. PLOS ONE 2015;10(9).

[22] Su CTT, Kwoh CK, Verma CS, Gan SKE. Modeling the full length HIV-1 Gag polyprotein reveals the role of its p6 subunit in viral maturation and the effect of non-cleavage site mutations in protease drug resistance. J Biomol Struct Dyn 2018;36:4366-77.

[23] Deshmukh L, Louis JM, Ghirlando R, Clore GM. Transient HIV-1 Gag-protease interactions revealed by paramagnetic NMR suggest origins of compensatory drug resistance mutations. PNAS 2016;113:12456-61.

[24] Bell NM, Lever AML. HIV Gag polyprotein: processing and early viral particle assembly. Trends Microbiol 2013;21:136-44.

[25] Franke EK, Yuan HEH, Luban J. Specific incorporation of cyclophilin a into HIV1 virions. Nature 1994;372(6504):359-62.

[26] Braaten D, Franke EK, Luban J. Cyclophilin A is required for an early step in the life cycle of human immunodeficiency virus type 1 before the initiation of reverse transcription. J Virol 1996;70:3551-60.

[27] Carnes SK, Sheehan JH, Aiken C. Inhibitors of the HIV-1 capsid, a target of opportunity. Curr Opin HIV AIDS 2018;13:359-65.

[28] Purdy MD et al. MicroED structures of HIV-1 Gag CTD-SP1 reveal binding interactions with the maturation inhibitor bevirimat. PNAS 2018;115:13258-63.

[29] Seclén E et al. High prevalence of natural polymorphisms in Gag (CA-SP1) associated with reduced response to Bevirimat, an HIV-1 maturation inhibitor. AIDS 2010;24(3):467-9.

[30] Verheyen J et al. High prevalence of bevirimat resistance mutations in protease inhibitor-resistant HIV isolates. Aids 2010;24(5):669-73.

[31] Marzinek JK, Huber RG, Bond PJ. Multiscale modelling and simulation of viruses. Curr Opin Struct Biol 2020;61:146-52. https://doi.org/10.1016/j. sbi.2019.12.019.

[32] Ayton GS, Noid WG, Voth G. Multiscale modeling of biomolecular systems: in serial and in parallel. Curr Opin Struct Biol 2007;17:192-8.

[33] Grime JMA, Voth GA. Early stages of the HIV-1 capsid protein lattice formation. Biophys J 2012;103:1774-83.

[34] Grime JMA et al. Coarse-grained simulation reveals key features of HIV-1 capsid self-assembly. Nat Commun 2016;7:11568.

[35] Perilla JR, Schulten K. Physical properties of the HIV-1 capsid from all-atom molecular dynamics simulations. Nat Commun 2017;8(1). https://doi.org/ $10.1038 /$ ncomms15959.

[36] Pak AJ et al. Immature HIV-1 lattice assembly dynamics are regulated by scaffolding from nucleic acid and the plasma membrane. Proc Natl Acad Sci USA 2017; 114:E10056-65.

[37] Pak AJ, Grime JMA, Yu A, Voth GA. Off-pathway assembly: a broad-spectrum mechanism of action for drugs that undermine controlled HIV-1 viral capsid formation. J Am Chem Soc 2019;141:10214-24.

[38] Liu C et al. Cyclophilin A stabilizes the HIV-1 capsid through a novel noncanonical binding site. Nat Commun 2016;7(1). https://doi.org/10.1038/ ncomms10714.

[39] Huang P-T et al. FEZ1 is recruited to a conserved cofactor site on capsid to promote HIV-1 trafficking. Cell Rep. 2019;28(9):2373-2385.e7.

[40] Dick Robert A et al. Inositol phosphates are assembly co-factors for HIV-1. Nature 2018;560(7719):509-12.

[41] Charlier L et al. Coarse-grained simulations of the HIV-1 matrix protein anchoring: revisiting its assembly on membrane domains. Biophys J 2014;106:577-85.

[42] Hill CP, Worthylake D, Bancroft DP, Christensen AM, Sundquist WI. Crystal structures of the trimeric human immunodeficiency virus type 1 matrix protein: implications for membrane association and assembly. PNAS 1996;93:3099-104.

[43] Schur FKM et al. An atomic model of HIV-1 capsid-SP1 reveals structures regulating assembly and maturation. Science (80-.) 2016;353:506-8.

[44] Alfadhli A, Barklis RL, Barklis E. HIV-1 matrix organizes as a hexamer of trimers on membranes containing phosphatidylinositol-(4,5)-bisphosphate. Virology 2009;387(2):466-72.

[45] Tang C, Ndassa Y, Summers MF. Structure of the N-terminal 283-residue fragment of the immature HIV-1 Gag polyprotein. Nat Struct Biol 2002:9:537-43.

[46] Deshmukh L, Ghirlando R, Clorea GM. Conformation and dynamics of the Gag polyprotein of the human immunodeficiency virus 1 studied by NMR spectroscopy. Proc. Natl. Acad. Sci. U.S.A. 2015;112:3374-9.
[47] Brugger B et al. The HIV lipidome: a raft with an unusual composition. Proc Natl Acad Sci 2006;103:2641-6.

[48] Mattei S, Glass B, Hagen WJH, Kräusslich HG, Briggs JAG. The structure and flexibility of conical HIV-1 capsids determined within intact virions. Science (80-.). 2016;354:1434-7.

[49] Pettit SC et al. The p2 domain of human immunodeficiency virus type $1 \mathrm{Gag}$ regulates sequential proteolytic processing and is required to produce fully infectious virions. J Virol 1994;68:8017-27.

[50] Wassenaar TA et al. Going backward: a flexible geometric approach to reverse transformation from coarse grained to atomistic models. J Chem Theory Comput 2014;10(2):676-90.

[51] Ono A, Ablan SD, Lockett SJ, Nagashima K, Freed EO. Phosphatidylinositol $(4,5)$ bisphosphate regulates HIV-1 Gag targeting to the plasma membrane. PNAS 2004; 101:14889-94.

[52] Saad JS et al. Structural basis for targeting HIV-1 Gag proteins to the plasma membrane for virus assembly. PNAS 2006;101:14889-94.

[53] Ono A, Freed EO. Plasma membrane rafts play a critical role in HIV-1 assembly and release. PNAS 2001;98:13925-30.

[54] Thali M et al. Functional association of cyclophilin a with HIV-1 virions. Nature 1994;372(6504):363-5.

[55] Gamble TR et al. Crystal structure of human cyclophilin A bound to the aminoterminal domain of HIV-1 capsid. Cell 1996;87(7):1285-94.

[56] Sokolskaja E, Sayah DM, Luban J. Target Cell Cyclophilin A Modulates Human Immunodeficiency Virus Type 1 Infectivity. J Virol 2004;78:12800-8.

[57] Yin L, Braaten D, Luban J. Human immunodeficiency virus type 1 replication is modulated by host cyclophilin A expression levels. J Virol 1998:72:6430-6.

[58] Olsson MHM, SØndergaard CR, Rostkowski M, Jensen JH. PROPKA3: Consistent treatment of internal and surface residues in empirical pKa predictions. J Chem Theory Comput 2011;7:525-37.

[59] Campbell S et al. Modulation of HIV-like particle assembly in vitro by inositol phosphates. Proc Natl Acad Sci USA 2001;98:10875-9.

[60] Munro JB et al. A conformational transition observed in single HIV-1 Gag molecules during in vitro assembly of virus-like particles. J Virol 2014;88:3577-85.

[61] Datta SAK et al. Interactions between HIV-1 Gag Molecules in Solution: An Inositol Phosphate-mediated Switch. J Mol Biol 2007;365:799-811.

[62] Lu M et al. Atomic-resolution structure of HIV-1 capsid tubes by magic-angle spinning NMR. Nat Struct Mol Biol 2020;27:863-9.

[63] Harrison RK, Stein RL. Substrate specificities of the peptidyl prolyl cis-trans isomerase activities of cyclophilin and FK-506 binding protein: evidence for the existence of a family of distinct enzymes. Biochemistry 1990;29 (16):3813-6.

[64] Ke H, Mayrose D, Cao W. Crystal structure of cyclophilin A complexed with substrate Ala-Pro suggests a solvent-assisted mechanism of cis-trans isomerization. PNAS 1993;90:3324-8.

[65] Yezid H, Konate K, Debaisieux S, Bonhoure A, Beaumelle B. Mechanism for HIV1 tat insertion into the endosome membrane. J Biol Chem 2009;284:22736-46.

[66] $\mathrm{Ni} \mathrm{T}$ et al. Intrinsic curvature of the HIV-1 CA hexamer underlies capsid topology and interaction with cyclophilin A. Nat Struct Mol Biol 2020;27:855-62.

[67] Zhao G et al. Mature HIV-1 capsid structure by cryo-electron microscopy and all-atom molecular dynamics. Nature 2013;497(7451):643-6.

[68] Ayton GS, Voth GA. Multiscale computer simulation of the immature HIV-1 virion. Biophys J 2010;99:2757-65.

[69] Goh BC et al. Atomic modeling of an immature retroviral lattice using molecular dynamics and mutagenesis. Structure 2015;23:1414-25.

[70] Codoñer FM et al. Gag-protease coevolution analyses define novel structural surfaces in the HIV-1 matrix and capsid involved in resistance to Protease Inhibitors. Sci Rep 2017;7(1). https://doi.org/10.1038/s41598-017-03260-4.

[71] R. Datir, et al. In Vivo Emergence of a Novel Protease Inhibitor Resistance Signature in HIV-1 Matrix. bioRxiv 865840 (2019). doi: 10.1101/865840.

[72] Callahan MK et al. Phosphatidylserine on HIV envelope is a cofactor for infection of monocytic cells. J Immunol 2003;170:4840-5.

[73] Zaitseva E et al. Fusion stage of HIV-1 entry depends on virus-induced cell surface exposure of phosphatidylserine. Cell Host Microbe 2017;22 (1):99-110.e7.

[74] Sali A, Blundell T. Comparative protein modelling by satisfaction of spatial restraints. J Mol Biol 1994;234:779-815.

[75] Shen M, Devos D, Melo F, Sali A. A composite score for predicting errors in protein structure models. Protein Sci 2006;15:1653-66.

[76] Ramachandran GN, Ramakrishnan C, Sasisekharan V. Stereochemistry of polypeptide chain configurations. J Mol Biol 1963;7:95-9.

[77] Monticelli L et al. The MARTINI coarse-grained force field: Extension to proteins. J Chem Theory Comput 2008;4:819-34.

[78] Periole X, Cavalli M, Marrink S, Ceruso MA. Combining an elastic network with a coarse-grained molecular force field: structure, dynamics, and intermolecular recognition. J Chem Theory Comput 2009;5:2531-43.

[79] DeLano W. The PyMOL molecular graphics system. (2002).

[80] Jo S, Lim JB, Klauda JB, Im W. CHARMM-GUI membrane builder for mixed bilayers and its application to yeast membranes. Biophys J 2009;97:50-8.

[81] Qi Y et al. CHARMM-GUI martini maker for coarse-grained simulations with the martini force field. J Chem Theory Comput 2015;11:4486-94.

[82] Bussi G, Donadio D, Parrinello M. Canonical sampling through velocity rescaling. J Chem Phys 2007;126:014101. 
[83] Parrinello M, Rahman A. Polymorphic transitions in single crystals: a new molecular dynamics method. J Appl Phys 1981;52:7182-90.

[84] Huang J, MacKerell AD. CHARMM36 all-atom additive protein force field: Validation based on comparison to NMR data. J Comput Chem 2013;34:2135-45.

[85] Kim S et al. CHARMM-GUI ligand reader and modeler for CHARMM force field generation of small molecules. J Comput Chem 2017;38:1879-86.

[86] Hoover WG. Canonical dynamics: equilibrium phase-space distributions. Phys Rev A 1985;31:1695-7.

[87] Nosé S. A molecular dynamics method for simulations in the canonical ensemble. Mol Phys 1984;52(2):255-68.
[88] Essmann U et al. A smooth particle mesh Ewald method. J Chem Phys 1995; $103: 8577$.

[89] Hess B, Bekker H, Berendsen HJC, Fraaije JGEM. LINCS: a linear constraint solver for molecular simulations. J. Comp. Chem. 1997;18:1463-72.

[90] Abraham MJ et al. Gromacs: High performance molecular simulations through multi-level parallelism from laptops to supercomputers. SoftwareX 2015;12:19-25.

[91] Baker NA, Sept D, Joseph S, Holst MJ, McCammon JA. Electrostatics of nanosystems: application to microtubules and the ribosome. PNAS 2001;98:10037-41.

[92] Daura X et al. Peptide folding: when simulation meets experiment. Angew Chem 1999;38:236-40. 